\title{
SPHERE ZIMPOL: Overview and performance simulation
}

Christian Thalmann ${ }^{a}$, Hans M. Schmid ${ }^{a}$, Anthony Boccaletti ${ }^{b}$, David Mouillet ${ }^{c}$, Kjetil Dohlen $^{d}$, Ronald Roelfsema ${ }^{e}$, Marcel Carbillet ${ }^{f}$, Daniel Gisler ${ }^{a}$, Jean-Luc Beuzit ${ }^{c}$, Markus Feldt $^{g}$, Raffaele Gratton ${ }^{h}$, Franco Joos ${ }^{a}$, Christoph U. Keller ${ }^{i}$, Jan Kragt ${ }^{e}$, Johan H. Pragt ${ }^{e}$, Pascal Puget ${ }^{c}$, Florence Rigal ${ }^{e}$, Frans Snik $^{i}$, Rens Waters ${ }^{j}$, François Wildi ${ }^{k}$

${ }^{a}$ Institute of Astronomy, ETH Zürich, 8092 Zürich, Switzerland;

${ }^{b}$ LESIA, Observatoire de Paris, 5 place J. Janssen, 92195 Meudon, France;

${ }^{c}$ LAOG, UMR5571, CNRS/Université J. Fourier, BP 53X, 38041 Grenoble cedex 9, France;

${ }^{d}$ LAM, UMR6110, CNRS/Université de Provence, 13388 Marseille cedex 13, France;

eNOVA-ASTRON, Oude Hoogeveensedijk 4, 7991 PD Dwingeloo, The Netherlands;

fUMR 6525 H. Fizeau, UNS/CNRS/OCA, Campus Valrose, 06108 Nice cedex 2, France;

${ }^{9}$ Max-Planck-Institut für Astronomie, Königstuhl 17, 69117 Heidelberg, Germany;

${ }^{h}$ INAF, Osservatorio Astronomico di Padova, 35122 Padova, Italy;

${ }^{i}$ Sterrekundig Instituut Utrecht, Princetonplein 5, 3584 CC Utrecht, The Netherlands;

${ }^{j}$ Astronomical Institute "Anton Pannekoek", 1098 SJ Amsterdam, The Netherlands;

${ }^{k}$ Observatoire Astronomique de l'Université de Genève, 1290 Sauverny, Switzerland

\begin{abstract}
The ESO planet finder instrument SPHERE will search for the polarimetric signature of the reflected light from extrasolar planets, using a VLT telescope, an extreme AO system (SAXO), a stellar coronagraph, and an imaging polarimeter (ZIMPOL). We present the design concept of the ZIMPOL instrument, a single-beam polarimeter that achieves very high polarimetric accuracy using fast polarization modulation and demodulating CCD detectors. Furthermore, we describe comprehensive performance simulations made with the CAOS problem-solving environment. We conclude that direct detection of Jupiter-sized planets in close orbit around the brightest nearby stars is achievable with imaging polarimetry, signal-switching calibration, and angular differential imaging.
\end{abstract}

Keywords: SPHERE, ZIMPOL, extrasolar planets, direct detection, imaging polarimetry, signal switching, angular differential imaging

\section{INTRODUCTION}

The detection of reflected light from an old (>1 Gyr) Jupiter-sized extra-solar planet has not yet been achieved due to the very high intensity contrast $\left(10^{-8}\right)$ and the small angular separation between the planet and its parent star. One goal of the ESO planet finder instrument SPHERE ${ }^{1}$ (Spectro-Polarimetric High-contrast Exoplanet REsearch) is to perform such a direct detection using a large telescope (VLT), an extreme AO system, a stellar coronagraph, and a sophisticated polarization imager. Near-infrared imagers will be used for spectral differential imaging, e.g. in the methane bands.

Light scattered off a gas planet's atmosphere becomes polarized in the process. This provides the possibility to detect a polarization signal from a planet among the unpolarized residual light halo of the coronagraphic, AO-corrected image of a bright star with a sufficiently powerful polarimeter. ${ }^{2}$ In SPHERE, polarimetry will be possible with the Zurich Imaging Polarimeter $\left(\mathrm{ZIMPOL}^{3-5}\right)$, an instrument concept based on fast (kHz) polarization modulation and demodulating CCD detectors. ZIMPOL has been proven to achieve polarimetric sensitivities of $10^{-5}$. 


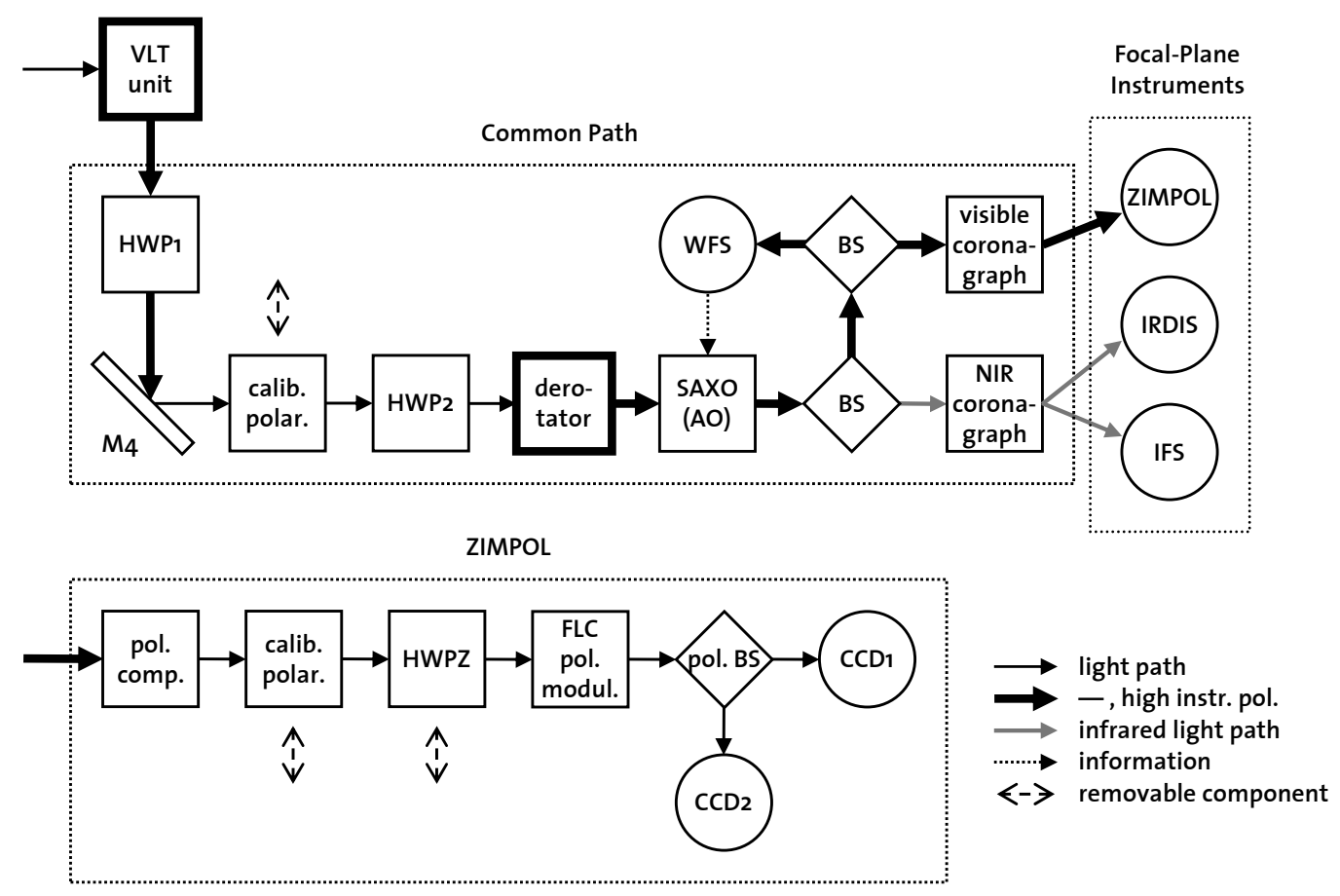

Figure 1. Schematic of the SPHERE instrument design, focusing on the components relevant to ZIMPOL. The components causing strong instrumental polarization (thick arrows) are marked with a thick outline. The mirror M4 and the polarization compensator (pol. comp.) are used to counteract these detrimental effects. Abbreviations: BS = beam splitter, HWP = half-wave plate, WFS = wave-front sensor, FLC = ferro-electric liquid crystal.

In order to investigate the feasibility of the required performance of this instrument (contrast $10^{-8}$ at a separation of $1^{\prime \prime}$ ), we provide simulations ${ }^{6}$ made with the software package SPHERE ${ }^{7}$ (a simulation code for $\mathrm{AO}$, diffraction, photometry and reduction developed within the CAOS problem-solving environment ${ }^{8}$ ), using up-to-date instrumental error estimations from the current SPHERE ZIMPOL design study as input parameters.

\section{INSTRUMENT OVERVIEW}

The SPHERE instrument is divided into four basic components: The three focal-plane scientific detectors IRDIS ${ }^{9}$ (InfraRed Dual-beam Imaging and Spectroscopy), IFS ${ }^{10}$ (Integral Field Spectrograph) and ZIMPOL, and the common path, an umbrella term for all other optics between the telescope interface and the scientific detectors. Figure 1 shows a block diagram of the SPHERE ZIMPOL branch.

\subsection{Common Path}

The light coming from the VLT unit telescope is afflicted with instrumental polarization from the off-axis mirror M3. A half-wave plate (HWP1) and a tiltable mirror (M4) are used to compensate this polarization offset. A set of polarizers can be inserted at this point for calibration purposes (calib. polar.).

Another half-wave plate (HWP2) mounted on a rotation stage can be used to reverse the sign of the (astronomical) polarization of the light beam, allowing for a convenient form of beam-exchange calibration during observations (subsequently referred to as "signal switching").

Further downstream, a three-mirror field derotator can physically reorient the coordinate system of the sky field image by a given angle. If this component is deactivated, the motion of the altitude-azimuth mounting of the VLT unit telescope while tracking a target on the continuously spinning globe of the sky will result in a rotation of the field image over time. The derotator can be activated to counteract the natural field rotation, resulting in a constant field orientation during the whole observation. Finally, to avoid smearing during long exposures 


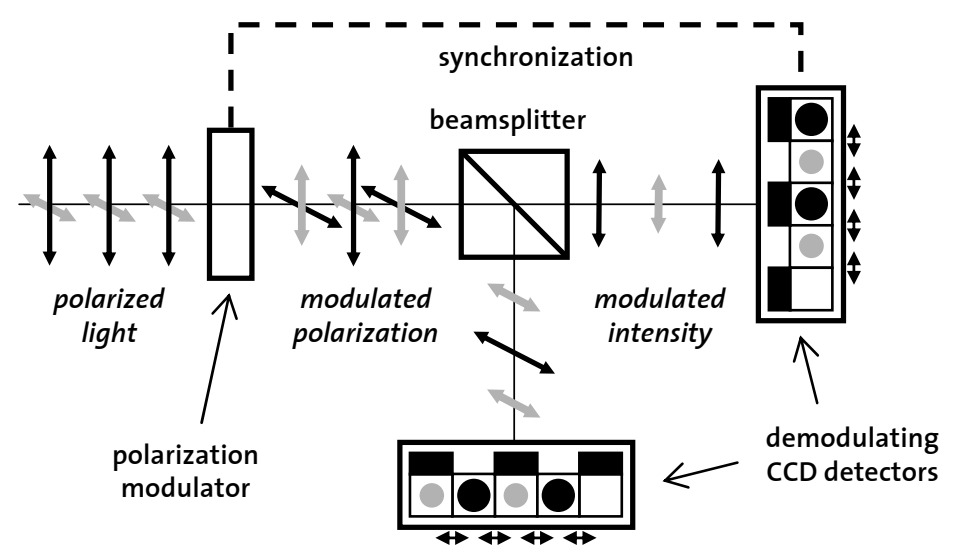

Figure 2. A schematic of the ZIMPOL detector concept. Note that a single CCD camera would suffice for a fully functional imaging polarimeter; the second camera merely ensures that no photons are wasted.

without field stabilization, the derotator can be set to compensate the differential field rotation during each single exposure of the science detector, but to reset to its starting position between exposures. The derotator allows the field orientation to be arbitrarily changed so as to facilitate angular differential imaging.

Then follow the various corrective components of the adaptive optics system SAXO (SPHERE Adaptive optics for eXoplanet Observation). It uses a deformable mirror of $180 \mathrm{~mm}$ diameter with $41 \times 41$ actuators with inter-actuator stroke $> \pm 3.5 \mu \mathrm{m}$, and a two-axis tip-tilt mirror with \pm 0.5 mas resolution. ${ }^{11}$ Further downstream in the visible branch of the optical path, a Shack-Hartman wavefront sensor (WFS) with $40 \times 40$ lenslets operating in the $0.45-0.95 \mu \mathrm{m}$ band at a temporal sampling frequency of $1.2 \mathrm{kHz}$ using a $240 \times 240$ pixel electron-multiplying CCD detector feeds the corrective optics with real-time information, enabling a global AO loop delay below 1 ms.

A dichroic beam splitter (BS) separates the visible from the near-infrared (NIR) part of the light, allowing the wavefront sensor to use the full visible flux during NIR observations. Further beamsplitters divide the beams between the WFS and ZIMPOL, and IRDIS and IFS, respectively.

Finally, the common path includes one full coronagraph assembly for each of the two spectral bands. Each features selection wheels that allow the focal mask and pupil stop to be chosen from a set of Lyot or fourquadrant phase mask (4QPM) components. The coronagraphs attenuate the central peak of the target star's image, greatly reducing the total brightness contrast in the image and thereby allowing longer frame exposure times, while at the same time purging the diffraction features caused by the propagated pupil edges from the image.

\subsection{ZIMPOL}

The first component of the ZIMPOL science instrument is a device for compensating the instrumental polarization introduced by components further upstream, in particular the field derotator. It consists of a glass plate that is tilted and rotated to produce an opposite polarization offset in the transmitted beam. The compensation is necessary to minimize the impact of detector nonlinearities. Then follow a set of calibration polarizers and a half-wave plate (HWPZ) with which the linear polarization can be aligned with the orientation of ZIMPOL. These two components are retractable.

The final four components form the ZIMPOL polarimeter per se. A ferro-electric liquid crystal (FLC) polarization modulator exchanges the two complementary polarization components of the light at up to $1 \mathrm{kHz}$, and a subsequent polarizing beamsplitter (pol. BS) translates the polarization-modulated beam into two intensitymodulated beams. Each beam is focused onto a specialized CCD camera which performs the demodulation in real-time on the sensor, using masked buffer pixel rows interlaced between the sensitive pixel rows to store one polarized image while the other is being exposed to light. The photocharges are shifted between the buffer rows and the sensitive rows in synchronization with the modulation. This way, the sensor can integrate both component images quasi-simultaneously over normal frame exposure times (e.g. one second), using the same detection pixels 


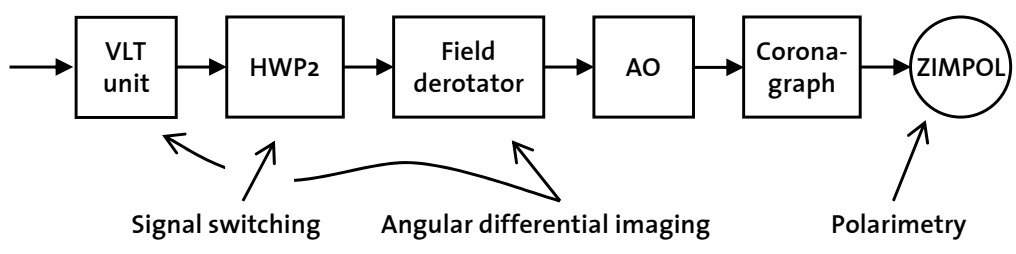

Figure 3. A simplified scheme of the SPHERE ZIMPOL instrument pointing out the key components for the three differential imaging techniques explained in Section 3. Angular differential imaging can be based on the "natural" field rotation caused by the telescope tracking, or on artificial "active" field rotation performed by the derotator optics.

for both images. The shifting of the charge map by one row is quick enough to allow for modulation frequencies in the $\mathrm{kHz}$ range. The speckle noise issue of the conventional single-beam setup is effectively defeated. Figure 2 illustrates this working principle.

Although there are two sensors, ZIMPOL is a single-beam design; each sensor acts as a fully functional imaging polarimeter. This redundancy can be exploited to calibrate residual instrumental effects, or to select two different areas of interest in the field, for instance.

While ZIMPOL is remarkably free of differential flatfielding and calibration issues, laboratory tests for highcontrast duty in SPHERE ${ }^{12}$ have revealed a more subtle weakness of the system: Polarized differential aberrations. Switching the FLC modulator between its two retardance states also slightly changes its other optical properties. A slight state-dependent wedge effect that affects the pointing of the transmitted beam ("differential pointing") as well as some higher-order phase-like differential aberrations have been found. Although these effects are very small (of the order $1 \mathrm{~nm}$ RMS), they constitute the primary limiting factor to the polarimetric precision of ZIMPOL in high-contrast applications.

The ZIMPOL detector of SPHERE is designed to work in the $V, R$ and $I$ bands and is optimized for 600$900 \mathrm{~nm}$. The field of view is a square of $3.5^{\prime \prime}$ that can be shifted around the target star within a field of $4^{\prime \prime}$ radius. A polarimetric accuracy of at least $10^{-5}$ has been attested to the system in laboratory tests, ${ }^{13}$ and $10^{-6}$ appears feasible.

Aside from the differential imaging polarimetry, the ZIMPOL camera will also provide high-resolution direct imaging in a variety of broad- and narrow-band visible filter ranges, filling the important niche of imaging circumstellar regions at high contrast and high angular resolution.

\section{DIFFERENTIAL TECHNIQUES}

In order to meet the extreme constrast requirements of direct planet imaging, the ZIMPOL branch of SPHERE employs several stages of differential imaging. The raw intensity image of the coronagraphically masked target star will be dominated by speckle-like noise caused by the static and quasi-static aberrations of the instrument, about four orders of magnitude above the level where a planet signal could be detected.

The ZIMPOL-specific requirements that instrumental polarization must be kept low and that measurements of the absolute degree of polarization must be possible impose restrictions on the optical design and therefore on the differential methods available. Figure 3 shows the components of the instrument responsible for each differential method.

Polarimetry. The most obvious differential technique employed in SPHERE ZIMPOL is imaging polarimetry: By taking an image of two complementary polarization states and subtracting them from each other, the unpolarized component of the image, including all the starlight, should be removed. In practice, polarized differential aberrations between the two polarization channels will limit the efficiency of this subtraction, leaving behind an artificial background landscape in the polarization image. Nevertheless, the $\mathrm{S} / \mathrm{N}$ gain brought about by polarimetry is on the order of $10^{2}$.

Signal switching. To calibrate away the spurious background in the polarization image, a second level of differential imaging is employed. By rotating a half-wave plate (HWP2) far upstream in the optical path by $45^{\circ}$, the sign of the incoming Stokes $Q$ polarization is reversed. The instrumental aberrations, on the other hand, 


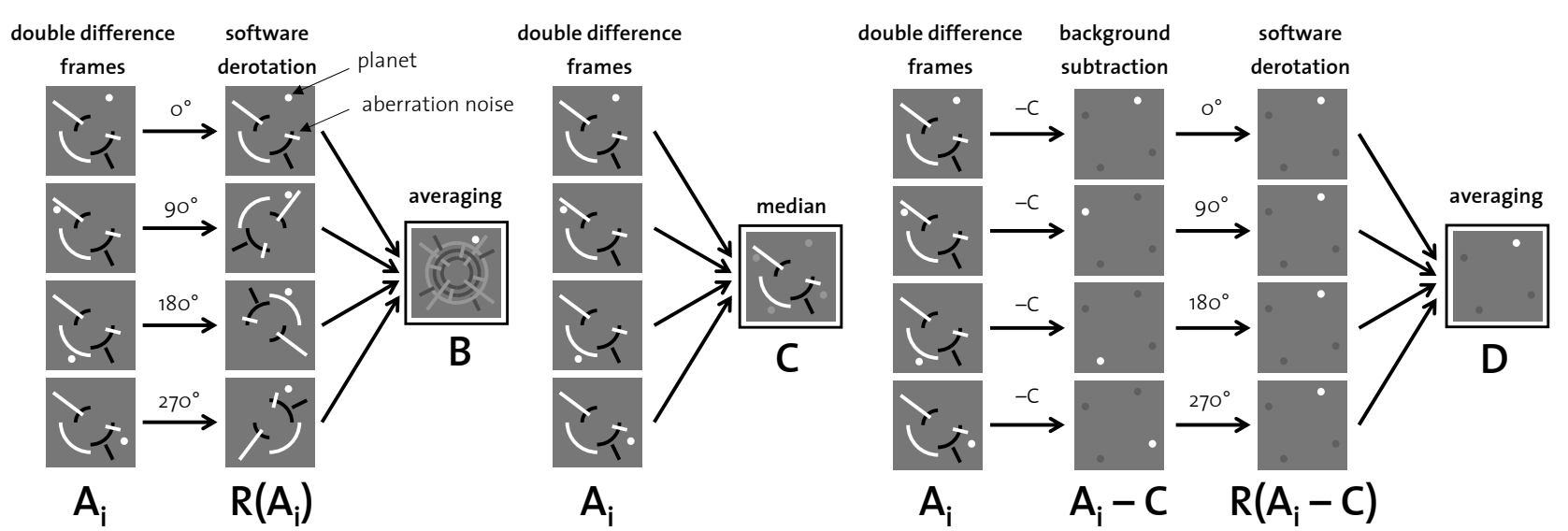

Figure 4. Schematic illustration of angular differential methods on the example of active field rotation with four positions. For angular averaging, the individual exposures $\left(A_{i}\right)$ are simply derotated into the coordinate system of the sky $\left(R\left(A_{i}\right)\right)$ and added up $(B)$, whereby the background structures are averaged down. For proper angular differential imaging with background subtraction, a median background of the raw exposures is first calculated $(C)$ and subtracted from each exposure $\left(A_{i}-C\right)$ before derotation $\left(R\left(A_{i}-C\right)\right)$ and final co-addition $(D)$.

remain unchanged, resulting in the same background landscape as before. If the polarization images before and after the signal switching are subtracted from one another, the real polarization signals of the astronomical target add up constructively while the static background is canceled out.

Again, this method is only effective down to the level at which the background can be reproduced in the second image; any change to the optical path on the time scale of the signal switching (i.e. temporal differential aberrations) will lead to residual structures surviving in the double-difference image, limiting the detectivity of faint signals. The half-wave plate is foreseen to be switched about every 5 minutes during observation.

This calibration method is comparable to the conventional subtraction of a reference PSF (taken from another star) from the target image; the half-wave plate switching is simply an elegant solution to obtaining a reference PSF with a minimum of differential effects and without a substantial amount of overhead time.

Angular differential imaging (ADI). A third level of noise suppression is achieved through angular differential imaging. Over the course of the observation, the VLT unit telescope's tracking will cause the observed sky field to rotate slowly with respect to the detector, whereas the optical elements responsible for aberrations will remain fixed in the instrument's frame of reference. This property can be exploited to discern the real signals originating in the sky from spurious instrumental effects through so-called angular differential imaging. ${ }^{14}$

A median average of all exposures of the observation run is calculated and subtracted from each individual exposure, removing all static parts of the image. Since the planet moves across the field in an arc during the observation, it does not leave a strong footprint in the median background and therefore survives the background subtraction. The exposures are then rotated into the sky's frame of reference such that the planet signals coincide and add up constructively, whereas any residual background noise is averaged down. This technique is illustrated in Figure 4 .

If the background evolves on the time scale of the field rotation, the background subtraction becomes ineffective. Nevertheless, the field rotation can be exploited for angular averaging, a less effective version of ADI.

In cases where the natural field rotation is insufficient (e.g. for a star near the celestial pole) or erratic (near the zenith), the image orientation on the detector can be artificially changed in discrete steps using the field derotator optics. This "active" field rotation extends the limited natural rotation arc to $2 \pi$. This is particularly beneficial in the center of the field, where the natural arc length is very limited.

With all three differential methods applied, the noise can likely be suppressed down to the statistical photon noise for a four-hour observation, as shown by the performance simulation presented below. 


\section{SIMULATION}

\subsection{Architecture}

To determine the feasibility of detecting exoplanets with SPHERE ZIMPOL and to investigate how its performance can be maximized, we have developed a detailed end-to-end performance simulation code. ${ }^{6,7}$ It is composed of two distinct programs.

The diffraction code produces point-spread functions (PSFs) both for the central star occluded by the coronagraph, and for the off-center planets. It is implemented in the IDL-based problem-solving environment $\mathrm{CAOS}^{8}$ as part of the software package SPHERE. The code simulates the effects of AO-corrected atmospheric turbulence and various static and differential aberrations. The output is saved to file.

The photometry and reduction code combines a set of PSFs provided by the diffraction code with numerical parameters describing properties of the target star, the planets, the observation procedure, the optical path and the detector, to simulate the final output pictures of an observation run. Differential imaging and optional signal enhancement methods are applied, and the resulting signal-to-noise curves are plotted to a PS file. This code is implemented as a free-standing IDL routine.

\subsection{Input parameters}

During the evolution of the SPHERE project, the accepted set of standard input parameters for the performance simulation has been updated regularly. The following tables provide an overview of the Standard Case 3 (SC3) as defined in Thalmann $(2008)^{15}$ and references therein. The differential techniques have been improved for SC4.

Atmospheric parameters

Seeing at $500 \mathrm{~nm}$

Turbulent Layers:

- altitude $z[\mathrm{~m}]$ :

$-C_{n}^{2}$ profile [\%]:

- wind speed $v[\mathrm{~m} / \mathrm{s}]$ :

- direction $\left[^{\circ}\right]$ :

AO guide star parameters

Magnitude:

Zenith angle $\left[^{\circ}\right]$ :

Azimuth angle $\left.{ }^{\circ}\right]$ :
Telescope parameters

Diameter [m]:

Obscuration ratio:

Zero point $\left[\mathrm{e}^{-} / \mathrm{m}^{2} \mathrm{~s}\right]$

0.14

$0 ; 1000 ; 10000$

$20 ; 60 ; 20$

$12.5 ; 12.5 ; 12.5$

$0 ; 45 ; 90$

AO system parameters

Imaging wavelength $[\mathrm{nm}]$

Linear \# sub-apertures:

Integration time [s]:

0.000833

Loop delay $[\mathrm{s}]$ :

0.001

Loop gain:

0.465999

WFS wavelength [nm]:

650

Read-out noise $\left[\mathrm{e}^{-}\right]$:

Dark current $\left[\mathrm{e}^{-} / \mathrm{s}\right]$ :

Spatial filter efficiency:

The AO performance is insensitive to the guide star brightness down to $\sim 8$ mag, where it starts to degrade due to photon noise in the WFS. For this reason, an arbitrary bright magnitude of 4 is chosen.

\section{Static Aberrations}

Telescope Mirror M1 [nm]:

Telescope Mirror M2 [nm]:

Telescope Mirror M3 [nm]:

Instrument [nm]:

AO calibration $[\mathrm{nm}]$ :

Fresnel propagation [nm]:

Beam shift [nm]:

\section{Temporal Aberrations}

Defocus [nm]:

Pupil shear [ $D$ unit]:

Pupil rotation $\left[^{\circ}\right.$ :

Differential rotation $\left[^{\circ}\right]$ :

Temporal beam shift [nm]:

Temporal high-freq. aberr. [nm]: $\quad 11$

0

Temporal pointing [mas]:

The temporal aberrations describe the changes and drifts in the common path on the time scale of the signal switching, which therefore cause differential effects between the target and reference channel, and limit the effectiveness of this calibration method. The half-wave plate responsible for signal switching (HWP2) is foreseen to toggle its state every few minutes in order to minimize these effects. For this reason, most parameters can 
be set to small values or zero. The imperfect surface of the half-wave plate itself, however, introduces its own temporal aberration whenever it rotates. While the low spatial frequencies of these aberrations are corrected by the AO, a high-frequency term remains (11 nm RMS in SC3, new goal $5.5 \mathrm{~nm}$ in SC4).

Coronagraph parameters

Mask radius $[\lambda / D$ at $600 \mathrm{~nm}]$ :

Pupil stop transmission [\%]:

Filter bandwidth [nm]:

Filter central wavelength $[\mathrm{nm}]$ :

Number of wavelengths simulated:

\section{ZIMPOL Aberrations}

ADC residuals [mas RMS]:

Phase differential aberr. [nm]:

Pointing differential aberr. [nm]:

Downstream static aberr. [nm]:

Beam shift $[\mathrm{nm}]$ :

Laboratory tests conducted at ASTRON and ETH Zürich ${ }^{12}$ have shown that the beams emerging from the FLC modulator in its two states are not exactly parallel. This differential pointing corresponds to a gradientshaped aberration of $1.5 \mathrm{~nm}$ RMS. Furthermore, an interferometer test revealed "phase-like" (higher-order) differential aberrations of $1 \mathrm{~nm}$ RMS. Finally, the differential pointing causes the outgoing beams to pass through slightly different crosssections of the subsequent optical surfaces, thus the static aberrations induced by the imperfections of those surfaces are not identical between the two polarizations. This so-called beam shift effect adds another $1.1 \mathrm{~nm}$ of phase-like differential aberrations. These three specifications are cut in half for SC4.

\section{Photometric parameters}

Star type:

Star distance $[\mathrm{pc}]$ :

Exposure time [h]:

Total transmission:

Full-well capacity [photons/px]:

Read-out noise [photons/px]:

\section{Planet parameters}

Angular separations ["]:

Size [Jupiter radii]:

Phase angle $\left[^{\circ}\right]$ :

Phase-dependent albedo:

Degree of polarization [\%]:
$0.05-0.5$

$10^{5}$

10

10 and 50

Here, $[\mathrm{px}]$ refers to a CCD hardware pixel. A pixel in the final reduced image, on the other hand, is implemented with 32 such hardware pixels ( 8 exposed and 8 masked pixels on each of 2 cameras).

Since a planet's PSF core extends over more than one pixel, convolving the final image with a correspondingly sized aperture removes all noise on the pixel-to-pixel scale while preserving the planet signal. We find that this specific form of low-pass filtering improves the $\mathrm{S} / \mathrm{N}$ ratio by a factor of at least 2 if the image is dominated by statistical photon noise. The method fails in aberration-dominated images, where the background landscape shares the planet's spatial scale. By default, we apply a Gaussian weighted convolution aperture with a FWHM of 2 pixels in all simulations.

\section{RESULTS}

\subsection{Standard Case Performance}

The detectivity curves for a simulation under Standard Case 3 conditions (G0V star at 3 pc, classical Lyot coronagraph, $4 \mathrm{~h}$ observation time) are presented in Figure 5. For visual reference, the two-dimensional detection images at each stage are shown. In the double-difference image (curve C), a spurious background landscape caused by the combination of static, polarized and temporal aberrations still obscures any planet signals. Angular differential imaging is necessary to reduce this background to a level where planets can be detected.

In the worst-case scenario of a double-difference background fluctuating on the time scale of field rotation, only angular averaging is possible. Curves D and E show the performance for active field rotation over 23 discrete positions and for natural field rotation over a continuous arc of $45^{\circ}$, respectively. In either case, the best-case scenario is a complete elimination of the aberration-dominated background, leaving only the photon noise (curve $\mathrm{F}$ ).

The detection zone for planets ranges from 0 ". 08 (the edge of the $5 \lambda / D$ coronagraph) to $0{ }^{\prime \prime} 3$ (the control radius of the $\mathrm{AO})$. The $\mathrm{S} / \mathrm{N}$ ratios improve with decreasing angular separation. At the photon limit, a $25 \%$ polarized Jupiter-sized planet is detectable at 0. . 3 with $\sim 5 \sigma$ confidence; at 0 "! 1 , the same planet reaches $\sim 50 \sigma$.

Note that a prolongation of the observation time by the factor $N$ will lower the photon limit by a factor of $1 / \sqrt{N}$. However, this gain can only be harnessed if all other noise sources are successfully suppressed below the photon noise level, which then presents an increasingly challenging task. 

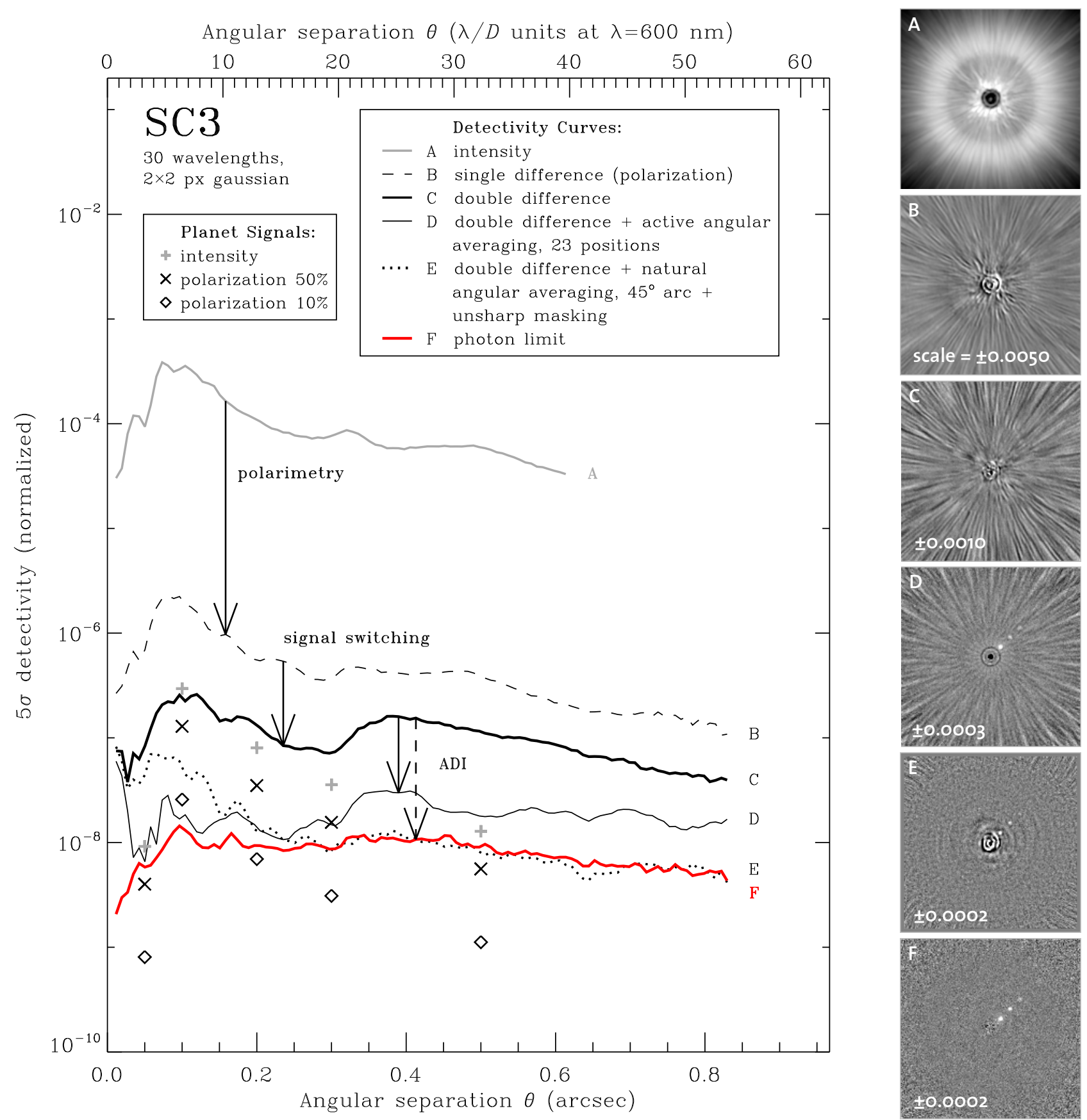

Figure 5. Left: Performance plot for a Standard Case 3 simulation (G0V star at 3 pc) using a Lyot coronagraph with $5 \lambda / D$ mask radius at $\lambda=600 \mathrm{~nm}$, after $4 \mathrm{~h}$ of observation. The detectivity curves show the level at which a signal is detected with a confidence of $5 \sigma$. The simulated broadband images were composed from 30 monochromatic PSFs, and convolved with a Gaussian aperture of $2 \mathrm{px}$ width in order to suppress noise below the spatial scale of planet signals. The planet at $\theta=0$ ". 05 is obscured by the coronagraphic mask. Angular averaging (D, E) is the worst-case performance of ADI; in the ideal case, the photon limit (F) is reached. Right: The images corresponding to the plotted curves. Aperture convolution was not applied here in order to preserve visual detail. 


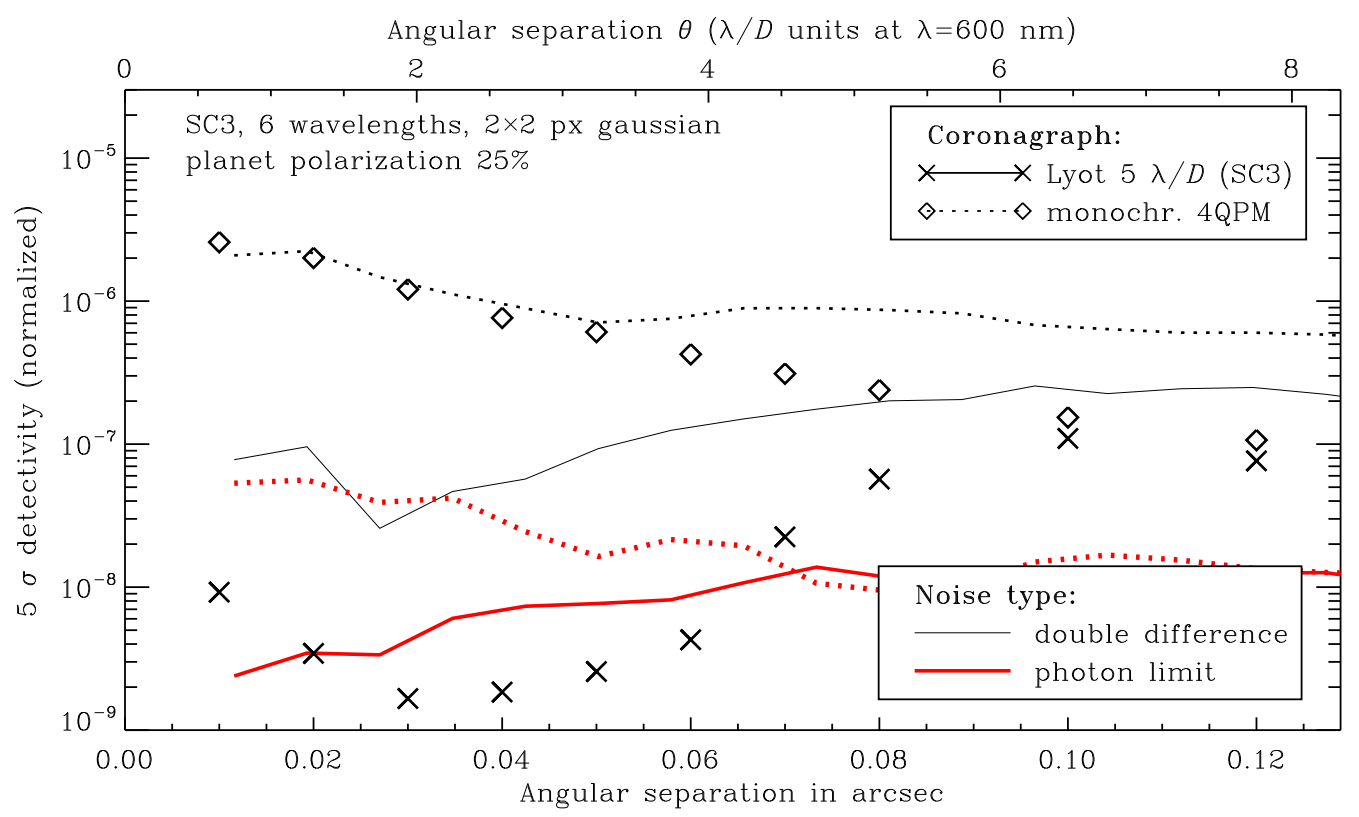

Figure 6. The double-difference (thin lines) and photon-limit performances (thick lines) of the $5 \lambda / D$ Lyot (solid lines) and monochromatic 4QPM coronagraphs (dotted lines) in the inner region of 0 " -0 ". 12 . The broadband range was sampled with 6 wavelengths. The symbols denote the signal of a Jupiter-sized planet with $25 \%$ polarization.

\subsection{Four-quadrant phase mask coronagraph}

Since the default $5 \lambda / D$ Lyot coronagraph obscures the innermost region of the field where the planet/star contrast is the most favorable, an alternative coronagraph based on the monochromatic four-quadrant phase mask design ${ }^{16}$ is foreseen for SPHERE ZIMPOL. While its rejection performance is inferior to the Lyot design, it allows the detection of sources as close as a single resolution element $(\lambda / D \approx 0$ !'015) from the target star. Figure 6 shows a comparison of the performances of the two coronagraph designs in the inner region of $00^{\prime \prime} 0$ ". 12 .

\subsection{Parameter dependences}

We have run a comprehensive analysis of the dependence of the simulated performance on the various input parameters. The most crucial four dependences are described below.

Target distance. The brightness contrast of planet and star is proportional to the inverse square of their physical distance. Therefore, the $\mathrm{S} / \mathrm{N}$ ratio in the aberration-dominated regime at a given angular separation scales with the inverse square of the distance to the target system, $1 / d^{2}$. In the photon-dominated regime, the detectivity curve is no longer constant, but scales with the inverse square root of the photon flux, sharpening the relation to $S / N \propto 1 / d^{3}$.

Target luminosity. In the aberration-dominated case, the performance is (by definition) insensitive to the luminosity $L$ of the target star. In the photon-limited case, the photon flux determines the noise level and therefore the detectivity: $S / N \propto \sqrt{L}$. This seemingly weak dependence is important due to the extreme differences in luminosity between A and M stars.

Polarized differential aberrations. The amplitude of the wedge-like and higher-order differential aberrations caused by the FLC modulator (see Section 4.2) directly scales the S/N ratio in the aberration-dominated doubledifference image. While the $\mathrm{S} / \mathrm{N}$ ratio in the photon-limit image is not affected, an improvement of the doubledifference image makes it much more likely that the photon limit can be reached with ADI. Thus, minimizing these effects in the modulator is a high-priority technical goal.

Temporal differential aberrations. The surface imperfections of the half-wave plate HWP2 translate into temporal differential aberrations when it is rotated for the purpose of signal switching. The AO can only compensate the low spatial frequencies of those aberrations. The $\mathrm{S} / \mathrm{N}$ ratio in the double-difference image 
Table 1. The most favorable target stars for SPHERE ZIMPOL in terms of detectivity. For each star, the absolute $V$-band magnitude and distance in parsecs is given, as well as the $\mathrm{S} / \mathrm{N}$ ratio for the detection of a $25 \%$ polarized Jupiter-sized planet at two angular separations $(0.03,0$.'2) and two different noise level assumptions (active angular averaging over 23 field orientations, photon limit), and the frame exposure time $t_{\exp }$ needed to reach $80 \%$ of full well capacity. The $5 \lambda / D$ classical Lyot coronagraph is assumed for the $\theta=0.2$ case, and the monochromatic 4QPM coronagraph for $\theta=0$ ! 03 . Otherwise, SC3 conditions are used. Integration time is 4 hours.

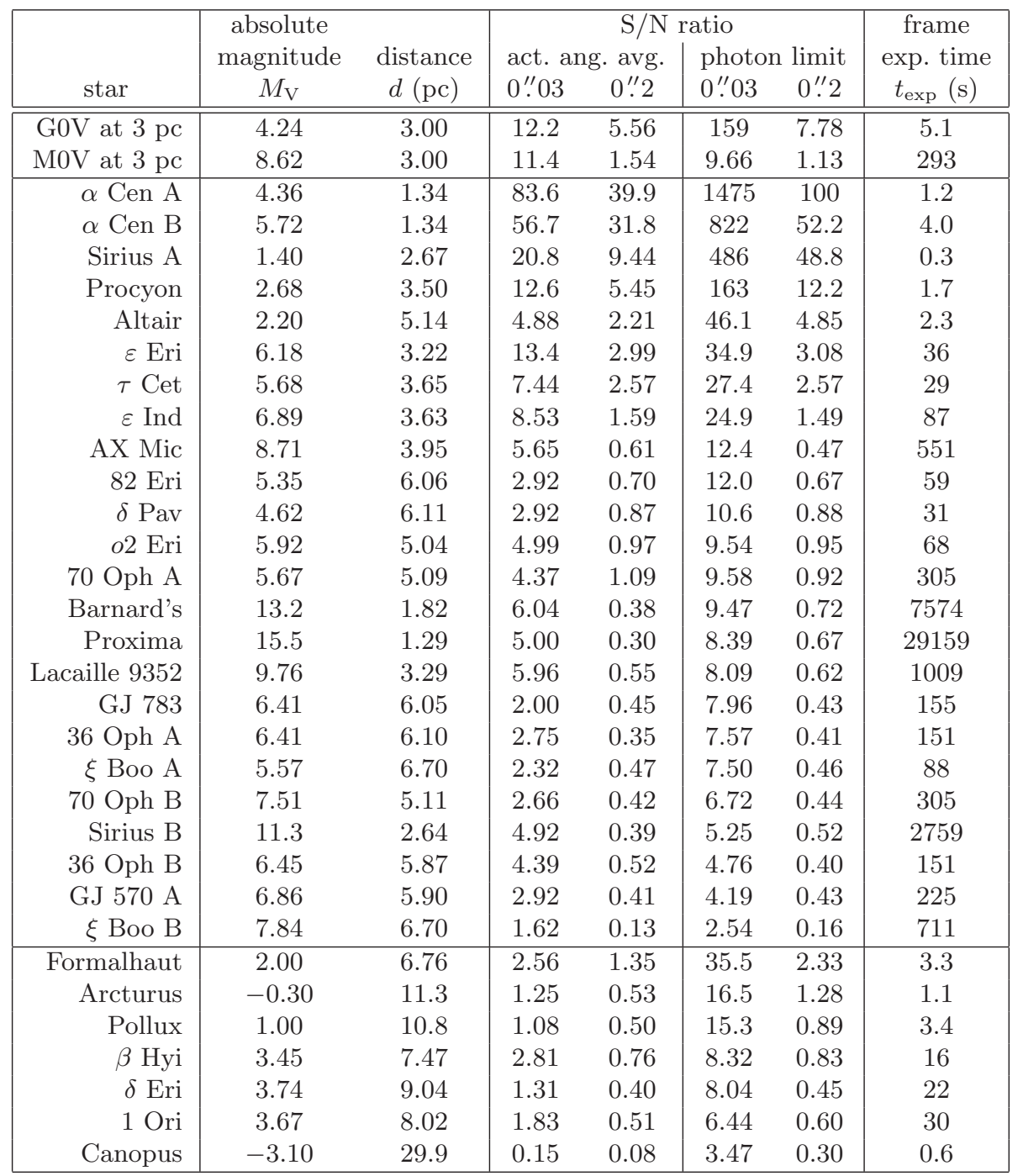

scales linearly with the amplitude of the remaining high-frequency terms. The surface roughness of HWP2 must therefore be kept as low as possible.

\subsection{Target stars}

The relations given in Section 5.3 imply that the $\mathrm{S} / \mathrm{N}$ ratio for planet detection drops rapidly with increasing target distance or decreasing target luminosity. Only the nearest and brightest stars are therefore promising candidates.

A list of the most favorable target stars and their $\mathrm{S} / \mathrm{N}$ ratios for $25 \%$ polarized Jupiter-sized planets at two different angular separations is given in Table 1, assuming a $4 \mathrm{~h}$ observation. The angular averaging is to be taken as the worst-case scenario for the effectiveness of ADI, whereas the photon limit marks the ideal case. The table shows that Jupiter-sized planets can in principle be detected within the AO control radius ( 0 "! 3$)$ around the 

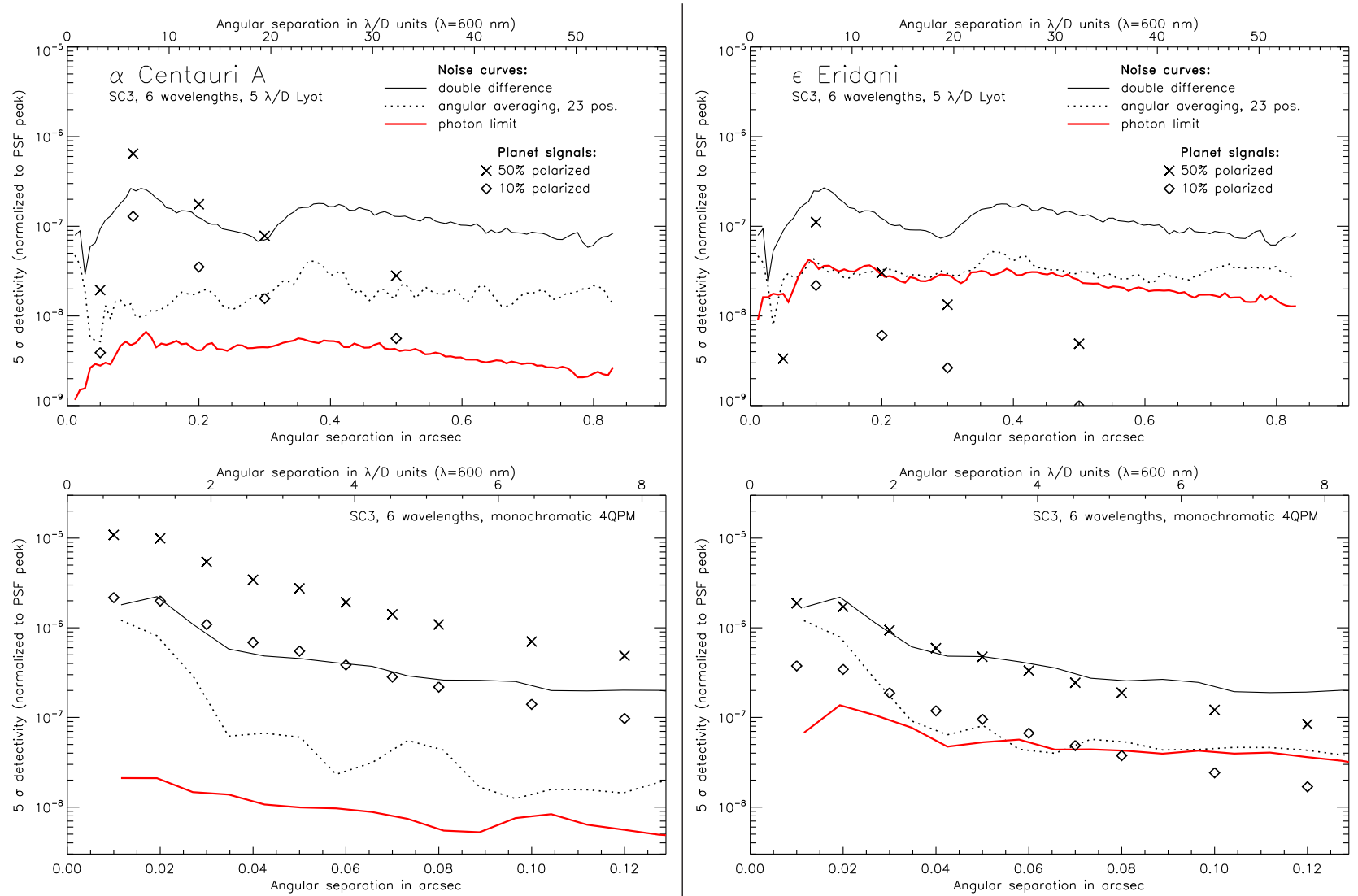

Figure 7. The performance plots for $\alpha$ Centauri A (left) and $\varepsilon$ Eridani (right), using the $5 \lambda / D$ (top) and 4QPM (bottom) coronagraphs. Otherwise, Standard Case 3 conditions are applied.

eight best target stars in a matter of a few nights using the $5 \lambda / D$ Lyot coronagraph. The 4QPM coronagraph allows detection of similar planets at very close separations $(<0$ ". 1$)$ around at least 20 stars. Due to the extreme dependence on target distance, even Neptune-sized planets ( $\mathrm{S} / \mathrm{N}$ reduced by a factor of $\sim 0.1$ ) or super-Earths ( $\sim 0.01$ ) could be found around the closest bright stars ( $\alpha$ Cen A and B, Sirius A etc.), and Jupiter-sized planets are detectable even outside the $\mathrm{AO}$ control radius.

Figure 7 presents the performance for two particular target stars, $\alpha$ Cen A and $\varepsilon$ Eri. The former is both closer and inherently brighter than the latter, resulting in stronger planet signals and lower photon noise. Note that the double-difference detectivity is insensitive to those astronomical parameters.

For its secondary science objects such as circumstellar disks, SPHERE ZIMPOL's range will extend much further. For example, the debris disk of TW Hydrae at a distance of $56 \mathrm{pc}$ will be visible in detail.

\section{CONCLUSION}

We have developed a comprehensive end-to-end performance simulation and analysis for SPHERE ZIMPOL. We find that the detection of exoplanets is feasible by combining three differential noise reduction techniques: High-precision imaging polarimetry, signal-switching calibration, and angular differential imaging. With the default $5 \lambda / D$ Lyot coronagraph, the candidate list for finding Jupiter-sized planets comprises 8 target stars. Furthermore, a monochromatic four-quadrant phase mask coronagraph grants access to the innermost 0 ". 1 of the field, extending the candidate list to over 20 stars. Even Neptune-sized planets or super-Earths could be detected around the best few target stars. Finally, we have identified the polarization modulator of ZIMPOL and the signal-switching half-wave plate as the most critical components; in response, the specifications for their differential behavior have been cut in half in order to improve the instrument performance. 


\section{REFERENCES}

[1] Beuzit, J.-L., Feldt, M., Dohlen, K., Mouillet, D., Puget, P., Antichi, J., Baruffolo, A., Baudoz, P., Berton, A., Boccaletti, A., Carbillet, M., Charton, J., Claudi, R., Downing, M., Feautrier, P., Fedrigo, E., Fusco, T., Gratton, R., Hubin, N., Kasper, M., Langlois, M., Moutou, C., Mugnier, L., Pragt, J., Rabou, P., Saisse, M., Schmid, H. M., Stadler, E., Turrato, M., Udry, S., Waters, R., and Wildi, F., "SPHERE: A 'Planet Finder' Instrument for the VLT," The Messenger 125, 29-34 (2006).

[2] Schmid, H. M., Beuzit, J.-L., Feldt, M., Gisler, D., Gratton, R., Henning, T., Joos, F., Kasper, M., Lenzen, R., Mouillet, D., Moutou, C., Quirrenbach, A., Stam, D. M., Thalmann, C., Tinbergen, J., Verinaud, C., Waters, R., and Wolstencroft, R., "Search and investigation of extra-solar planets with polarimetry," IAU Colloq. 200, 165-170 (2006).

[3] Povel, H. P., Aebersold, F., and Stenflo, J., "Charge-coupled device image sensor as a demodulator in a 2-D polarimeter with a piezoelastic modulator," Appl. Opt. 29, 1186 (1990).

[4] Povel, H. P., Keller, C., and Yadigaroglu, I.-A., "Two-dimensional polarimeter with a charge-coupled-device image sensor and a piezo-elastic modulator," Appl. Opt. 33, 4254 (1994).

[5] Povel, H., "Imaging stokes polarimetry with piezoelastic modulators and charge-coupled-device image sensors," Optical Engineering 34, 1870 (1995).

[6] Thalmann, C., Applications of High-Precision Polarimetry to Extrasolar Planet Search and Solar Physics, $\mathrm{PhD}$ thesis, ETH Zürich (Switzerland) (2008).

[7] Carbillet, M., Boccaletti, A., Thalmann, C., Fusco, T., Vigan, A., Mouillet, D., and Dohlen, K., "The software package SPHERE: a numerical tool for end-to-end simulations of the VLT instrument SPHERE," Proc. SPIE $\mathbf{7 0 1 5}$ (2008).

[8] Carbillet, M., Verinaud, C., Guarracino, M., Fini, L., Lardiere, O., Le Roux, B., Puglisi, A. T., Femenia, B., Riccardi, A., Anconelli, B., Correia, S., Bertero, M., and Boccacci, P., "CAOS: a numerical simulation tool for astronomical adaptive optics (and beyond)," Proc. SPIE 5490, 637-648 (2004).

[9] Dohlen, K., Langlois, M., and Saisse, M., "The infra red dual imaging and spectrograph for SPHERE: design and performance," Proc. SPIE 7014 (2008).

[10] Claudi, R., Turatto, M., Gratton, R., Antichi, J., Bruno, P., Cascone, E., De Caprio, V., Desidera, S., Mesa, D., and Scuderi, S., "SPHERE IFS: the spectro differential imager of the VLT for exoplanets search," Proc. SPIE 7014 (2008).

[11] Petit, C., Fusco, T., Conan, J.-M., Sauvage, J.-F., Rousset, G., Gigan, P., Charton, J., Mouillet, D., Rabou, P., Kasper, M. E., Fedrigo, E., Hubin, N., Feautrier, P., Beuzit, J.-L., and Puget, P., "The SPHERE XAO system: design and performance," Proc. SPIE 7015 (2008).

[12] Roelfsema, R. and Gisler, D., "ZIMPOL FLC Test Report," ESO internal document VLT-TRE-SPH-146900185, ASTRON (2007).

[13] Thalmann, C., Testing the ZIMPOL Concept for Exoplanet Search, Master's thesis, ETH Zürich (2004).

[14] Marois, C., Lafrenière, D., Doyon, R., Macintosh, B., and Nadeau, D., "Angular Differential Imaging: A Powerful High-Contrast Imaging Technique," ApJ 641, 556-564 (2006).

[15] Thalmann, C., "Zimpol simulations and performance analysis report," ESO internal document VLT-TRESPH-14690-0191 issue 2, ETHZ (2008).

[16] Rouan, D., Riaud, P., Boccaletti, A., Clénet, Y., and Labeyrie, A., "The Four-Quadrant Phase-Mask Coronagraph. I. Principle," PASP 112, 1479-1486 (2000). 